\title{
Predictive Accuracy of Total IgE in Detection of Inhalant Allergens Sensitization
}

\author{
Moufag M. S. Tayeb ${ }^{1, ~ *, ~ M a j d y ~ M . ~ Q u t u b ~}{ }^{2}$ \\ ${ }^{1}$ Family Medicine, Faculty of Medicine, Jeddah University, Jeddah, Saudi Arabia \\ ${ }^{2}$ Family Medicine, Faculty of Medicine, King Abdulaziz University, Jeddah, Saudi Arabia
}

\section{Email address:}

moufagta@yahoo.com (M. M. S. Tayeb),mmqutub@kau.edu.sa (M. M. Qutub)

${ }^{*}$ Corresponding author

\section{To cite this article:}

Moufag M. S. Tayeb, Majdy M. Qutub. Predictive Accuracy of Total IgE in Detection of Inhalant Allergens Sensitization. Journal of Family Medicine and Health Care. Vol. 2, No. 1, 2016, pp. 6-9. doi: 10.11648/j.jfmhc.20160201.12

Received: March 11, 2016; Accepted: March 30, 2016; Published: April 25, 2016

\begin{abstract}
Background: The role of total IgE (TIGE) in detecting inhalant allergens (IA) sensitization in asthmatics is controversial and frequently underestimated. Objective: To test the relationship between TIGE and IA sensitization in asthmatics. Methods: One year duration cross-sectional study commencing from January in 2014. 118 asthmatics with mean age of $30 \pm 15$ years collected from King Abdulaziz University Hospital (KAUH). TIGE and skin prick test (SPT) was done. Data were statistically analyzed by statistical package for the social sciences program (SPSS). Two by two tables was done to correlate TIGE and IAs sensitization. Sensitivity, specificity, positive predictive values (+PV) and negative predictive values (PV) were calculated. Results: SPSS two by two tables was used to correlate TIGE with IA sensitization by sensitivity, specificity and predictive values. TIGE versus mites showed high sensitivity: Dermatophagoides pteronyssinus (DP) 80.3, Dermatophagoides farina (DF) 83.7 and low (specificity, +PV and -PV). TIGE versus mold showed high -PV to (Aspergillus 82.1, Cladosporium 78.5) and low (sensitivity, specificity and +PV). TIGE versus German cockroach showed high sensitivity 90 and high -PV 92.8, however both specificity and +PV were low. All the values were statistically significant with P values below 0.05. Conclusion: Raised TIGE in asthmatics is of value in predicting dust mites and cockroach sensitization and exclusion of mold sensitization. High TIGE in asthmatics almost certainly mean the presence of mite sensitization. Conversely, normal TIGE in asthmatics can exclude mold sensitization. TIGE have both roles with German cockroach (highly sensitive, high $-\mathrm{PV})$.
\end{abstract}

Keywords: Allergy, Total IgE, Inhalant Allergen, Sensitization, Atopy, Skin Prick Test

\section{Introduction}

Humoral immunity start from $\mathrm{T}$ helper lymphocytes number two (Th2). Th2 cells are activated by cytokines secreted from CD4. Th2 lymphocytes are the main cells responsible about B lymphocyte induction to produce immunoglobulins (Igs) in response to a specific triggering agent. Igs are mainly IgG, $\operatorname{IgM}, \operatorname{IgE}$ and $\operatorname{IgD}$. [1]

$\operatorname{IgE}$ is responsible about allergic type I hypersensitivity reaction. It lands over mast cells after the first exposure to allergen in a process called sensitization. With re-exposure to the same allergen mast cells explode and produce a lot of inflammatory mediators which could induce allergic cascade. The allergical symptoms related these organs can appear in the eyes, nose, sinuses, lung and skin. [2]

Immunoglobulin $\mathrm{E}$ (IgE) is of two types: specific $\operatorname{IgE}$ (sIgE) against a specific allergen and TIGE which is the sum of all sIgEs against all allergens. SIgE can be tested in two ways: in vivo skin prick test (SPT) or in vitro radioallergosorbent blood test (RAST). High TIGE give a general clue about the presence of allergy, however positive $\mathrm{sIgE}$ is more useful than TIGE because it determines the causative allergen. [3]

Asthma can be divided into allergic asthma (AA) and nonallergic asthma (NAA). AA is the type which is triggered by a specific allergen while NAA isn't. Thus, the detection of sIgEs or high TIGE in asthmatics is suggestive for AA. After diagnosing the causative allergens which trigger AA, it's a 
crucial step to educate the patients about ways of its avoidance as a step in AA management. [4]

In asthmatics, it's an evidence practice to test the sIgEs of the IAs to discover the causative allergen. However, measuring TIGE routinely in asthmatics to expect IA sensitization is still a debatable subject. Several international asthma guidelines either ignore the role of TIGE in IA diagnosis in asthmatics or state that it's a useless diagnostic tool. Nevertheless, other studies indicate that TIGE is of diagnostic help in asthmatics if other biomarkers are positive. [5]

Up to our knowledge, after searching for evidence it's an assumed suggestion that the link between TIGE and IAs in asthmatics was not done before.

\section{Methodology}

One year period cross-sectional study started from January in 2014. 118 asthmatics both adults and children were selected from King Abdulaziz University Hospital (KAUH) in Jeddah. Local research ethics committee at KAUH approved study protocol. In vitro TIGE and in vivo SPT was done. Data were loaded and statistically analyzed by SPSS program. Two by two tables was done to discover the connection between TIGE and IAs sensitization. Sensitivity, specificity, +PV and -PV was calculated. $\mathrm{P}$ values less than 0.05 was accepted as a statistically significant.

In vivo SPT was done for the following IAs: mold like aspergillus, fusarium, penicillium, candida, cladosporium and alternaria alternata. Mites like: DP and DF. German cockroach. Pollens like: mesquite, amaranthus retro, mimosa, oleaceae, timothy, bermuda, rye, mugwort, plantain, salsola kali, chenopodium album and date palm.

In vivo SPT results were checked on the forearm after 15 minutes of pricks. Patients were asked to quit oral antihistamines and steroids several days before the test. Positive control is histamine hydrochloride while phenolated glycerol-saline is the negative control. Positive sensitization to allergens is diagnosed if the wheal is $\geq 3 \mathrm{~mm}$ diameter.

\section{Results}

Results of plotting TIGE versus IAs gives an important results as follow: for mite it shows high sensitivity, low (specificity and predictive values). For mold it displays a high $-\mathrm{PV}$, low (sensitivity, specificity and $+\mathrm{PV}$ ). For German cockroach it illustrates both high sensitivity and -PV, specificity and $+\mathrm{PV}$ were low.

Dermatophagoides Pteronyssinus (DP) sensitivity was 80.3, while specificity value is 39.1 , +PV was 59.4 and -PV is 64.2. Dermatophagoides farina (DF) sensitivity level is 83.7, while specificity is $38.9,+\mathrm{PV}$ is 52.1 and $-\mathrm{PV}$ is 75 . The readings were considered as significant because $P$ value was 0.029 and 0.012 for DP and DF respectively (table 1).

Nevertheless, TIGE show a high -PV to mold as follow: Aspergillus 82.1, Cladosporium 78.5. Sensitivity, specificity and +PV of TIGE to mold are low. All the values are considered as significant with $\mathrm{P}$ values as $0.020,0.007$ for Aspergillus and Cladosporium correspondingly (table 1).

However, TIGE value in detecting German cockroach sensitization display a double pictures as a high (sensitivity and -PV). Sensitivity of TIGE to German cockroach is 90, while the $-\mathrm{PV}$ is 92.8 . Both specificity and $+\mathrm{PV}$ were low. Values are statistically significant because $\mathrm{P}$ value is 0.029 (table 1).

\section{Discussion}

TIGE is of worth in approving mite and in excluding mold sensitization in asthmatics, however with German cockroach TIGE have both roles at the same time. Elevated TIGE in asthmatics is of high sensitivity for mites, thus if asthma case is associated with high TIGE this could indicate the presence of sensitization to mites and it's a logical rationale to test for sIgEs against mites (table 2).

In contrast, normal TIGE is of high -PV to mold, therefore if TIGE is normal it's a logical approach to exclude mold sensitization if sIgE test against mold is negative. TIGE with German cockroach have both roles (high sensitivity and high -PV) which means the high possibility of the presence of sensitization to it with high TIGE plus its exclusion with normal TIGE (table 2).

Routine TIGE in vitro test in each asthmatic is still a controversial issue. National and international asthma guidelines which don't mention TIGE test in asthmatics are: Canadian, [6] New Zealand, [7] British Thoracic Society (BTS) [8] and Saudi Initiative for Asthma (SINA) guidelines. [9] Additionally, other guidelines which have illustrated that TIGE is useless in asthmatics are: Global Initiative of Asthma guideline (GINA) [10] which demonstrate that TIGE has no value as a diagnostic test for atopy. World Allergy Organization Guideline (WAO) [11] demonstrate that TIGE is not recommended for screening in asthmatics.

However, other guidelines which have stated that TIGE is of some help in asthmatics are: National Asthma Education and Prevention Program guideline (NAEPP) [12] which display that higher caregiver stress was significantly associated with high TIGE and atopic immune profile. Moreover, the American Academy of Allergy, Asthma \& Immunology (AAAAI) [13] guideline indicate that sum of TIGE plus sIgE positive tests may represent an efficient diagnostic tool in asthmatics.

However, there are a plenty of other evidences which could indicate that TIGE and asthma are related. Peter J study clarify that TIGE is higher in asthmatics with positive sIgE (at least 1 allergen), [14] while Hema Satwani found that raised TIGE can be considered as a predictor of allergy in asthmatic children. [15] Moreover, Cissy B conclude that TIGE is higher in asthmatic child under five years with eosinophilia. [16] Even asthmatic elderly patients have higher TIGE than non-asthmatics. [17]

Duane L correlate TIGE in asthmatic child with early sensitization and wheeze. This study established that TIGE is higher in early sensitized children and in persistent wheezer 
children. However, TIGE is highest in children who have both of the two factors (early sensitization and wheeze). [18]

Furthermore, TIGE is related to airway hyperactivity (AWH). M.R. Sears study elucidate that TIGE is high in asymptomatic children with AWH. Moreover, this study express that TIGE is higher in asthmatic children when compared to the non-asthmatic ones. [19] Additionally, another study display that there is a strong association between high TIGE and bronchial hyperactivity (BHR) in offspring of asthmatic parents. [20]

Likewise, TIGE is related to asthma control and severity. significantly correlated with uncontrolled Asthma, [21] while Koshak E study approve that the higher TIGE the greater asthma severity will be. [22] TENOR Study emphasize that a higher TIGE is associated with more severe or difficult to treat asthma. [23]

Raised TIGE level have several uses in asthmatics. High TIGE can be used as a marker for atopy if associated with positive SPT, [24] high blood eosinophil count and mast cells in nasal smear. [25] Additionally, TIGE can be used to detect the response to specific immunotherapy (SIT) in asthmatics because SIT may decrease TIGE in $36 \%$ of cases. [26] Furthermore, sIgE/TIGE ratio can be used as a predictor of Maneechotesuwan et al study confirmed that higher TIGE is

clinical response to SIT and when to stop it. [27] Lastly, high TIGE can be used to measure the dose of omalizumab given to allergic asthma. [28]

TIGE can be used to differentiate between allergic and non-allergic asthma in conjunction with other biomarkers. Koshak E study reveal that phadiatop and TIGE can be utilized as a complementary tool in the identification of AA. [29] Tollerud DJ study determine that TIGE is considered as one of the phenotypic markers of atopy in asthmatic's if associated with positive SPT or high eosinophilia. [30]

\section{Conclusion}

Elevated TIGE in asthmatics almost certainly mean the presence of mite sensitization because TIGE is highly sensitive to mites. Conversely, normal TIGE in asthmatics could exclude mold sensitization because TIGE have high PV to mold. TIGE have both roles with German cockroach (highly sensitive and high -PV). In asthmatics, in spite TIGE is controversial; it has a possible role as a cheap crude screening device for optimal diagnosis of IAs sensitization.

\section{Addendum}

Table 1. Correlation of high TIGE to inhalants sensitization.

\begin{tabular}{|c|c|c|c|c|c|}
\hline & Sensitivity & Specificity & Positive predictive value & Negative predictive value & P value \\
\hline Dermatophagoides Pteronyssinus & 80.3 & 39.1 & 59.4 & 64.2 & 0.029 \\
\hline Dermatophagoides Farina & 83.7 & 38.9 & 52.1 & 75 & 0.012 \\
\hline Cockroach G & 90 & 33.7 & 26 & 92.8 & 0.029 \\
\hline Aspergillus & 28.9 & 25.5 & 2.8 & 82.1 & 0.020 \\
\hline Cladosporium & 25 & 24.7 & 2.8 & 78.5 & 0.007 \\
\hline
\end{tabular}

Table 2. The meanings of normal and high TIGE.

\begin{tabular}{|c|c|c|c|c|}
\hline \multirow[b]{2}{*}{ Inhalant Allergens } & \multicolumn{2}{|l|}{ High TIGE } & \multicolumn{2}{|l|}{ Normal TIGE } \\
\hline & Could Indicate Sensitization & $\begin{array}{l}\text { Don't Indicate } \\
\text { Sensitization }\end{array}$ & Almost Exclude Sensitization & $\begin{array}{l}\text { Don't Exclude } \\
\text { Sensitization }\end{array}$ \\
\hline Dermatophagoides Pteronyssinus & $\sqrt{ }$ & & & $\sqrt{ }$ \\
\hline Dermatophagoides Farina & $\sqrt{ }$ & & & $\sqrt{ }$ \\
\hline Cockroach G & $\sqrt{ }$ & & & $\sqrt{ }$ \\
\hline Aspergillus & & $\sqrt{ }$ & $\sqrt{ }$ & \\
\hline Cladosporium & & $\sqrt{ }$ & $\sqrt{ }$ & \\
\hline
\end{tabular}

\section{References}

[1] Abbas A, Lichtman A, Pallai S. Basic Immunology: Humoral Immune Response. $4^{\text {th }}$ ed. 2014: 131.

[2] Abbas A, Lichtman A, Pallai S. Basic Immunology: hypersensitivity. $4^{\text {th }}$ ed. 2014: 209.

[3] Cots P, Pena JM, Botey J, Eseverri JL, Marín A, Ras R. Determination of total and specific IgE using UNICAP 100: comparative study with the CAP system. Allergol Immunopathol (Madr). 1998.

[4] Romanet-Manent S, Charpin D, Magnan A, Lanteaume A, Vervloet D. Allergic vs. nonallergic asthma: what makes the difference?. Allergy. Jul 2002.
[5] D Sinclair, S A Peters. The predictive value of total serum IgE for a positive allergen specific IgE result. J Clin Pathol. Sep 2004.

[6] M Diane Lougheed, et al. Canadian Thoracic Society Guideline Update: Diagnosis and Management of Asthma in Preschoolers, Children and Adults: Executive Summery. 2012. ASTHMA. New Zealand Asthma Guideline. Sep 2002.

[8] British Guideline on the Management of Asthma (Quick Reference Guide). SIGN: Scottish Intercollegiate Guidelines Network. 2014.

[9] Guidelines for the diagnosis and management of asthma in adults and children. SINA: The Saudi Initiative for Asthma. 2016.
[7] THE DIAGNOSIS AND TREATMENT OF ADULT 
[10] POCKET GUIDE FOR ASTHMA MANAGEMENT AND PREVENTION (for adults and children older than 5 years). GINA: Global Initiative For Asthma. 2015.

[11] S. G. O. Johansson, Tari Haahtela. World Allergy Organization Guidelines for Prevention of Allergy and Allergic Asthma. Int Arch Allergy Immunol. 2004.

[12] National Asthma Education and Prevention Program. Guidelines for the Diagnosis and Management of AsthmaUpdate on Selected Topics. 2002.

[13] James T. Li. et al. Attaining optimal asthma control: A practice parameter. J ALLERGY CLIN IMMUNOL. 2005.

[14] Gergen PJ, Arbes SJ Jr, Calatroni A, Mitchell HE, Zeldin DC.Total IgE levels and asthma prevalence in the US population: results from the National Health and Nutrition Examination Survey 2005-2006. J Allergy Clin Immunol. Sep 2009.

[15] Satwani H, Rehman A, Ashraf S, Hassan A. Is serum total IgE levels a good predictor of allergies in children?. J Pak Med Assoc. oct 2009.

[16] Kartasamita CB, Rosmayudi O, Demedts M. Total serum IgE and eosinophil count in children with and without a history of asthma, wheezing, or atopy in an urban community in Indonesia. The Respiratory Disease Working Group. J Allergy Clin Immunol. Dec 1994.

[17] King MJ, Bukantz SC, Phillips S, Mohapatra SS, Tamulis T, Lockey RF. Serum total $\operatorname{IgE}$ and specific $\operatorname{IgE}$ to Dermatophagoides pteronyssinus, but not eosinophil cationic protein, are more likely to be elevated in elderly asthmatic patients. Allergy Asthma Proc. Sep 2004.

[18] Duane L. Sherrill, Renato T Stein, Marilyn Halonen, Fernando D. Martinez. Total serum IgE and its association with asthma symptoms and allergic sensitization among children. JOURNAL OF ALLERGY AND CLINICAL IMMUNOLOGY. AUG 1999.

[19] Sears MR, Burrows B, Flannery EM, Herbison GP, Hewitt CJ, Holdaway MD. Relation between airway responsiveness and serum IgE in children with asthma and in apparently normal children. N Engl J Med. Oct 1991.

[20] Jianfeng $\mathrm{Xu}$. et al. Major Genes Regulating Total Serum Immunoglobulin E Levels in Families with Asthma. Am J Hum Genet. Nov 2000.
[21] Kittipong Maneechotesuwan, Pimtira Sujaritwongsanon, Tasneeya Suthamsmai. IgE production in allergic asthmatic patients with different asthma control status. JOURNAL OF THE MEDICAL ASSOCIATION OF THAILAND = CHOTMAIHET THANGPHAET. JANUARY 2010.

[22] Emad Koshak. Do IN VITRO IgE TESTS HAVE A ROLE IN IDENTIFYING ATOPIC ASTHMA? Current Allergy \& Clinical Immunology. March 2006.

[23] Haselkorn T, et al. Allergy, total serum immunoglobulin E, and airflow in children and adolescents in TENOR. Pediatr Allergy Immunol. Dec 2010.

[24] Kumar R, Singh BP, Srivastava P, Sridhara S, Arora N, Gaur SN. Relevance of serum IgE estimation in allergic bronchial asthma with special reference to food allergy. Asian Pac J Allergy Immunol. Dec 2006.

[25] Kajosaari M, Saarinen UM. Evaluation of laboratory tests in childhood allergy. Total serum IgE, blood eosinophilia and eosinophil and mast cells in nasal mucosa of 178 children aged 3 years. Allergy. Jul 1981.

[26] Ahmad Al Obaidi AH, Mohamed Al Samarai AG, Yahya Al Samarai AK, Al Janabi JM. The predictive value of IgE as biomarker in asthma. J Asthma. Oct 2008.

[27] Gulbin Bingol Karakoc, Mustafa Yilmaz, Derya Ufuk Altıntaş, and Seval Güneşer Kendirli. Can Serum-Specific IgE/Total IgE Ratio Predict Clinical Response to AllergenSpecific Immunotherapy in Children Monosensitized to House Dust Mite? Journal of Allergy. 2012.

[28] Normansell R, Walker S, Milan SJ, Walters EH, Nair P. Omalizumab for asthma in adults and children. Cochrane Database Syst Rev. Jan 2014.

[29] Emad Koshak. Do IN VITRO IgE TESTS HAVE A ROLE IN IDENTIFYING ATOPIC ASTHMA? Current Allergy \& Clinical Immunology. March 2006.

[30] David J Tollerud, George T O'connor, David Sparrow, Scott T Weiss. Asthma, Hay Fever, and Phlegm Production Associated with Distinct Patterns of Allergy Skin Test Reactivity, Eosinophilia, and Serum IgE Levels: The Normative Aging Study. American Review of Respiratory Disease. 1991. 